\title{
Supplementary Information \\ Cationic lignin-based hyperbranched polymers to circumvent drug resistance in Pseudomonas Keratitis
}

Pei Lin Chee a, Cally Owh ${ }^{\text {a }}$, Mayandi Venkatesh ${ }^{\mathrm{b}}$, Mercy Halleluyah Periayah ${ }^{\mathrm{b}}$, Zheng Zhang

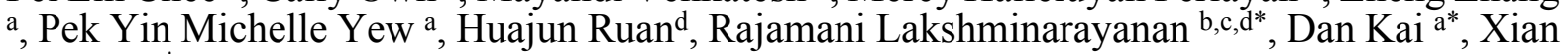
Jun Loh ${ }^{a^{*}}$

a. Institute of Materials Research and Engineering (IMRE), A*STAR, 2 Fusionopolis Way, \#08-03 Innovis, Singapore 138634.

b. Ocular Infections \& Anti-Microbials Research Group, Singapore Eye Research Institute, The Academia, 20 College Road, Discovery Tower, Singapore 169856.

c. Department of Pharmacy, National University of Singapore, 18 Science Drive, Singapore 117543.

d. Zhejiang Fenix Health Science and Technology Co., Ltd

e. Ophthalmology and Visual Sciences Academic Clinical Program, Duke-NUS Graduate Medical School, Singapore 169857

Number of pages: 9

Number of figures: 3

Number of tables: 2 


\section{Materials}

All chemicals were purchased from Sigma-Aldrich Chemicals and used as received except where noted. Alkali lignin $(\mathrm{Mn}=5,000 \mathrm{Da})$ was dried at $105^{\circ} \mathrm{C}$ overnight before use. N,Ndimethylaminoethyl methacrylate (DMAEMA) was purified by passing through a column with inhibitor remover before use.

\section{Synthesis of cationic lignin-based hyperbranched polymers}

The copolymers were synthesized in two steps as shown in Scheme 1. First, lignin-Br was obtained as follow: Dried lignin ( $3.0 \mathrm{~g}, 0.6 \mathrm{mmol}$, contains - OH $22.3 \mathrm{mmol}$ ) was weighed into a round bottle flask. Subsequently, anhydrous N,N-dimethylacetamide $(30 \mathrm{ml})$ with triethylamine (TEA, $53.5 \mathrm{mmol}, 7.46 \mathrm{ml}$ ) was added into the flask to dissolve the lignin under rapid stirring and $\mathrm{N}_{2}$ atmosphere. Then 2-bromoisobutyryl bromide (BIBB, 44.6 mmol, 5.51 ml) was added dropwise into the lignin solution under rapid stirring during a period of $1 \mathrm{~h}$ in ice-water bath. The reaction mixture was continued to stir for 1 day at room temperature. After that, the reaction mixture was centrifuged and the supernatant was precipitated with $500 \mathrm{ml}$ of ether. The tan gel-like precipitate was re-dissolved into tetrahydrofuran (THF, $50 \mathrm{ml}$ ) and the solution was then precipitated with $600 \mathrm{ml}$ of ether. The brown powder of lignin macroinitiator was collected and dried under vacuum at $40^{\circ} \mathrm{C}$.
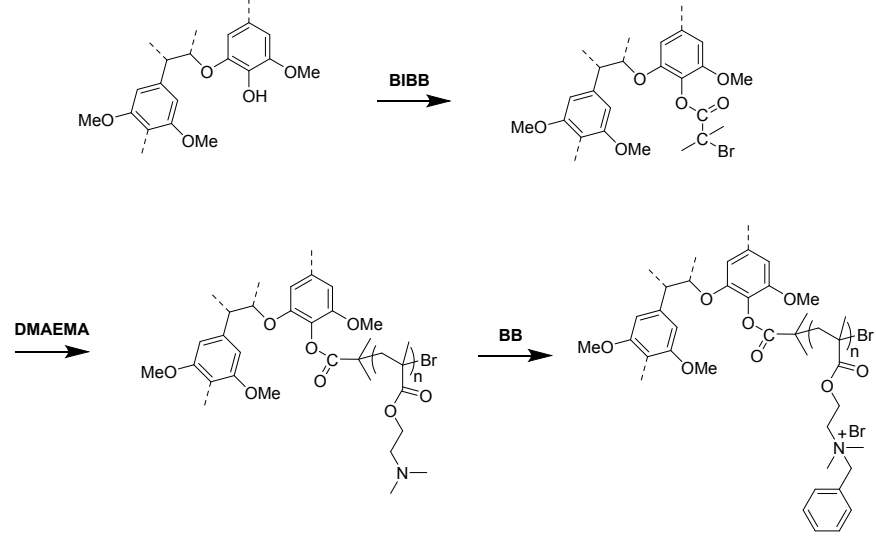

Scheme S1 Route to synthesis and quaternization of lignin-PDMAEMA copolymers 
Next, lignin-Br (130 mg, $0.3 \quad$ mmol Br $), \quad$ DMAEMA, 1,1,4,7,10,10Hexamethyltriethylenetetramine $(83 \mathrm{mg}, 0.36 \mathrm{mmol})$ and $15 \mathrm{ml}$ of degassed acetone were added into a dry flask. The mixture was stirred at room temperature and purged with dry nitrogen for $20 \mathrm{~min}$. After that, $\mathrm{CuBr}(43 \mathrm{mg}, 0.3 \mathrm{mmol})$ was added and the mixture was purged with dry nitrogen for another $5 \mathrm{~min}$ at room temperature. The mixture was continued to stir for overnight at room temperature. After that, the experiment was stopped by opening the flask and exposing the catalyst to air. The final tan mixture was diluted with tetrahydrofuran (THF) and passed through a short neutral $\mathrm{Al}_{2} \mathrm{O}_{3}$ column with THF as eluent to remove copper catalyst. The resulting solution was concentrated to $20 \mathrm{ml}$ and precipitated with $1000 \mathrm{ml}$ hexane. The brown product was collected by centrifugation, washed with hexane and dried under vacuum at $40{ }^{\circ} \mathrm{C}$. A series of copolymers with different compositions of the cationic polymer were prepared under similar conditions, as shown in Table 1.

\section{Characterization of cationic lignin-based hyperbranched polymers}

The copolymers were characterized by ${ }^{1} \mathrm{H}$ NMR (Bruker $400 \mathrm{MHz}$ ). Deuterated acetone or deuterium oxide was used as a solvent to dissolve the copolymers. The composition of the copolymers was obtained by using Elemental Analyser Flash 2000. The standards used were sulphanilimide to ensure that there is no error in the system. X-ray photoelectron spectroscopy (XPS) analysis was carried out using Thermo Fisher Scientific Theta Probe XPS with monochromatic Al Ka X-ray (1486.7 eV). Charge correction for binding energy was based on C1s from adventitious carbon at $285.0 \mathrm{eV}$.

The molecular weights and polydispersity indexes of the polymer samples were analysed by Waters gel permeation chromatography (GPC, a Shimadzu SCL-10A and LC-8A system equipped with two Phenogel $5 \mathrm{~mm} 50$ and $1000 \AA \AA$ columns in series and a Shimadzu RID-10A refractive index detector). HPLC-grade THF was used as an eluent. The flow rate of THF eluent 
was $1.0 \mathrm{~mL} / \mathrm{min}$ at $25^{\circ} \mathrm{C}$. The number average molecular weights $\left(\mathrm{M}_{\mathrm{n}}\right)$, weight average molecular weights $\left(M_{w}\right)$ and polydispersity index $\left(P D I, M_{w} / M_{n}\right)$ were determined with a calibration based on linear poly(methyl methacrylate) standards.

Thermal behaviors of the copolymers were investigated. Thermogravimetric analysis (TGA) was carried out on a thermogravimetric analyser (Q500, TA Instruments, USA). Samples ( 5 mg) were heated at $20{ }^{\circ} \mathrm{C} / \mathrm{min}$ from room temperature to $700{ }^{\circ} \mathrm{C}$ in a dynamic nitrogen atmosphere (flow rate $=60 \mathrm{ml} / \mathrm{min}$ ). Differential Scanning Calorimeter (DSC) thermal analysis was performed on a DSC (Q100, TA Instruments, USA) equipped with an autocool accessory and calibrated using indium. The following protocol was used for each sample: heating from $20{ }^{\circ} \mathrm{C}$ to $+180{ }^{\circ} \mathrm{C}$ at $20{ }^{\circ} \mathrm{C} / \mathrm{min}$, holding at $+180{ }^{\circ} \mathrm{C}$ from $5 \mathrm{~min}$, cooling from $+180{ }^{\circ} \mathrm{C}$ to -20 ${ }^{\circ} \mathrm{C}$ at $20{ }^{\circ} \mathrm{C} / \mathrm{min}$, and finally reheating from -20 to $+180{ }^{\circ} \mathrm{C}$ at $20{ }^{\circ} \mathrm{C} / \mathrm{min}$. Data were collected during the second heating run.

Particle size and zeta potential of the quaternized lignin copolymers were measured using the zetasizer device (Nano Z, Malvern, UK). The copolymers were prepared in DI water at a concentration of $1 \mathrm{mg} / \mathrm{ml}$.

\section{Quaternization of Lignin copolymers}

All lignin copolymers were quaternized to quaternary amine trimethylaminoethyl methacrylate (as shown in Scheme 1). $2 \mathrm{~g}$ of each copolymer was dissolved in THF by stirring and sonication. After that, certain amount of benzyl bromide (BB) was added into the copolymer solution at room temperature. Lignin copolymers were partially or fully quaternized by adding different amounts of BB (50 $\mu 1$ for partially and $200 \mu$ f fully). The mixture was continued to stir for 24 hours at $60{ }^{\circ} \mathrm{C}$. Then, the final mixture was precipitated with $50 \mathrm{ml}$ hexane and the product was collected by centrifugation, washed with hexane and dried under vacuum at $40{ }^{\circ} \mathrm{C}$. 
Table S1: Summary of the atomic ratio in LD30, LD50 and LD100 attained from XPS

\begin{tabular}{cccc}
\hline Elements/Composition (At\%) & LD30 & LD50 & LD100 \\
\hline $\mathrm{Br}$ & 0.2 & 0.2 & 0.1 \\
$\mathbf{C}$ & 73.4 & 74.2 & 73.7 \\
\hline $\mathbf{N}$ & 5.0 & 7.4 & 7.5 \\
\hline $\mathbf{O}$ & 21.3 & 18.3 & 18.7 \\
\hline
\end{tabular}


Table S2: Elemental weight analysis of nitrogen, carbon, hydrogen and sulphur atoms for LD30, LD50 and LD100

\begin{tabular}{cccc}
\hline Element Name & LD30 & LD50 & LD100 \\
\hline Nitrogen & 5.226 & 8.334 & 8.426 \\
\hline Carbon & 45.0667 & 59.9056 & 59.27 \\
\hline Hydrogen & 6.8154 & 9.3376 & 9.278 \\
\hline Totals & 57.1081 & 77.5772 & 76.974 \\
\hline
\end{tabular}


(A)

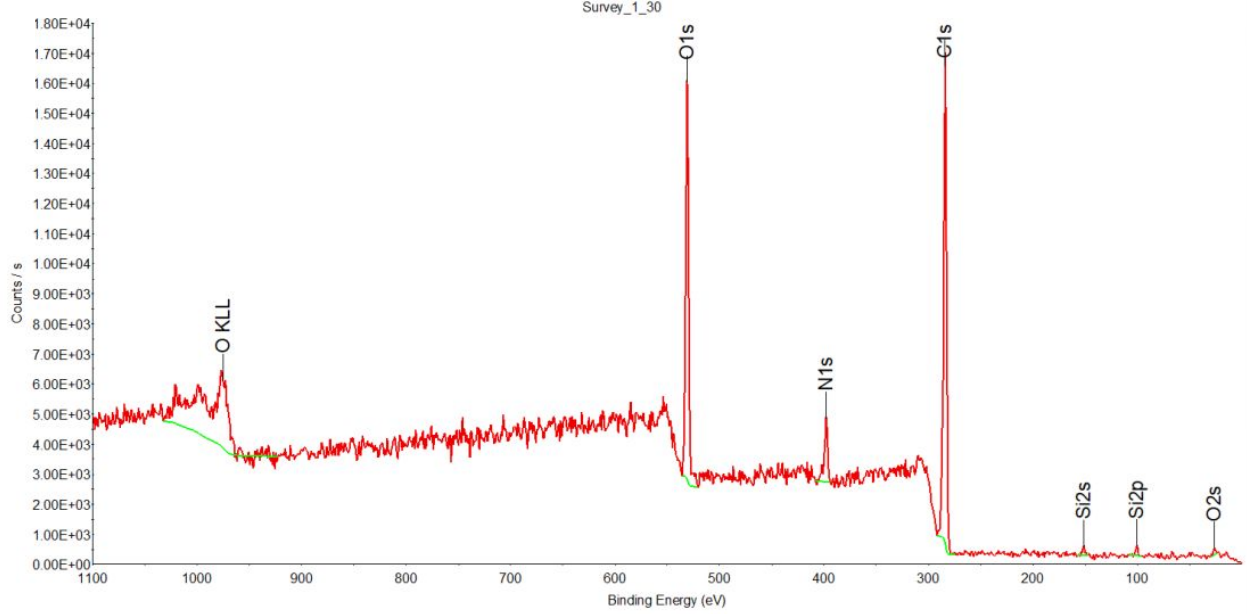

(B)

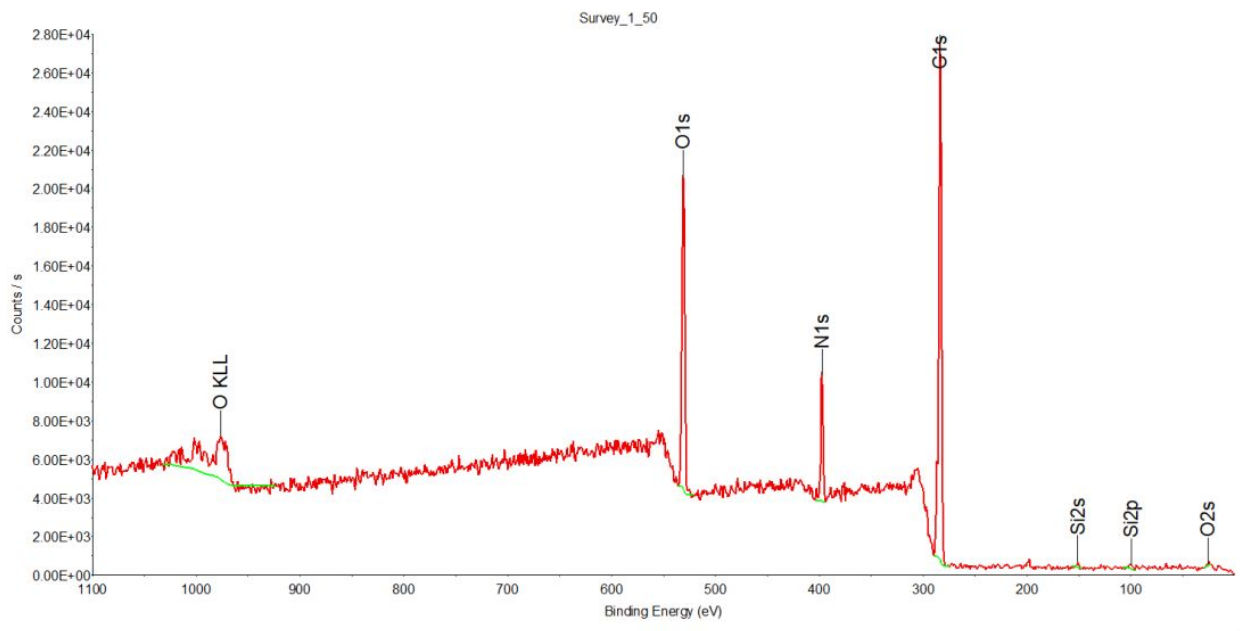

(C)

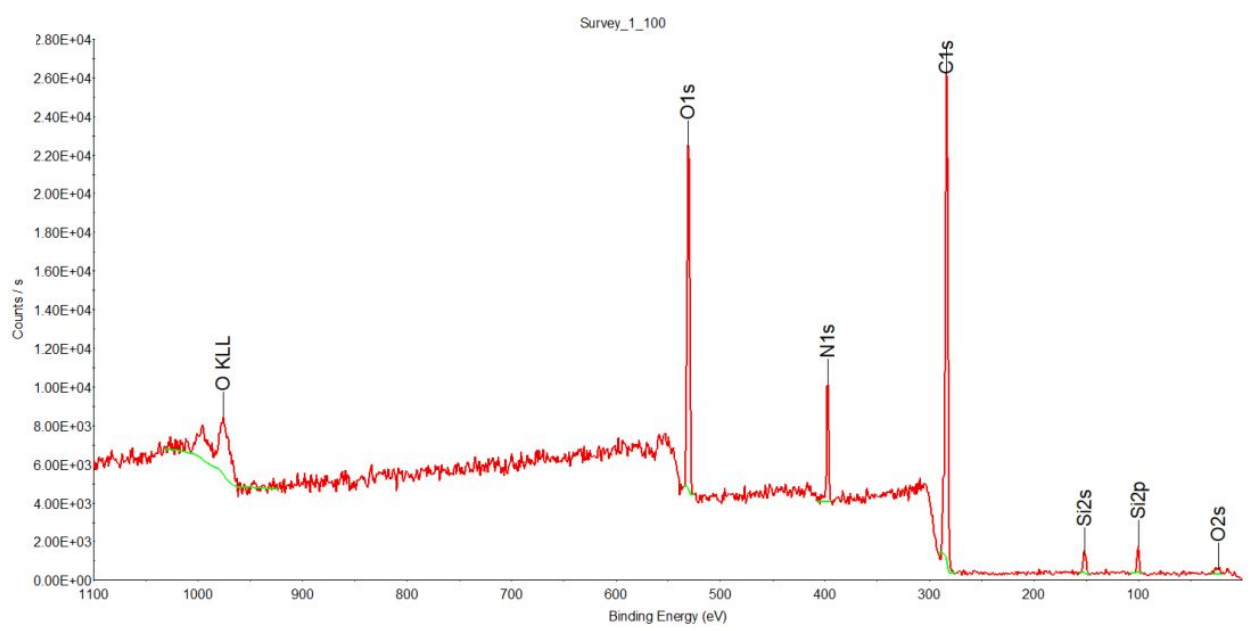

Figure S1: XPS chart representation of LD30 (A), LD50 (B) and LD100 (C) 


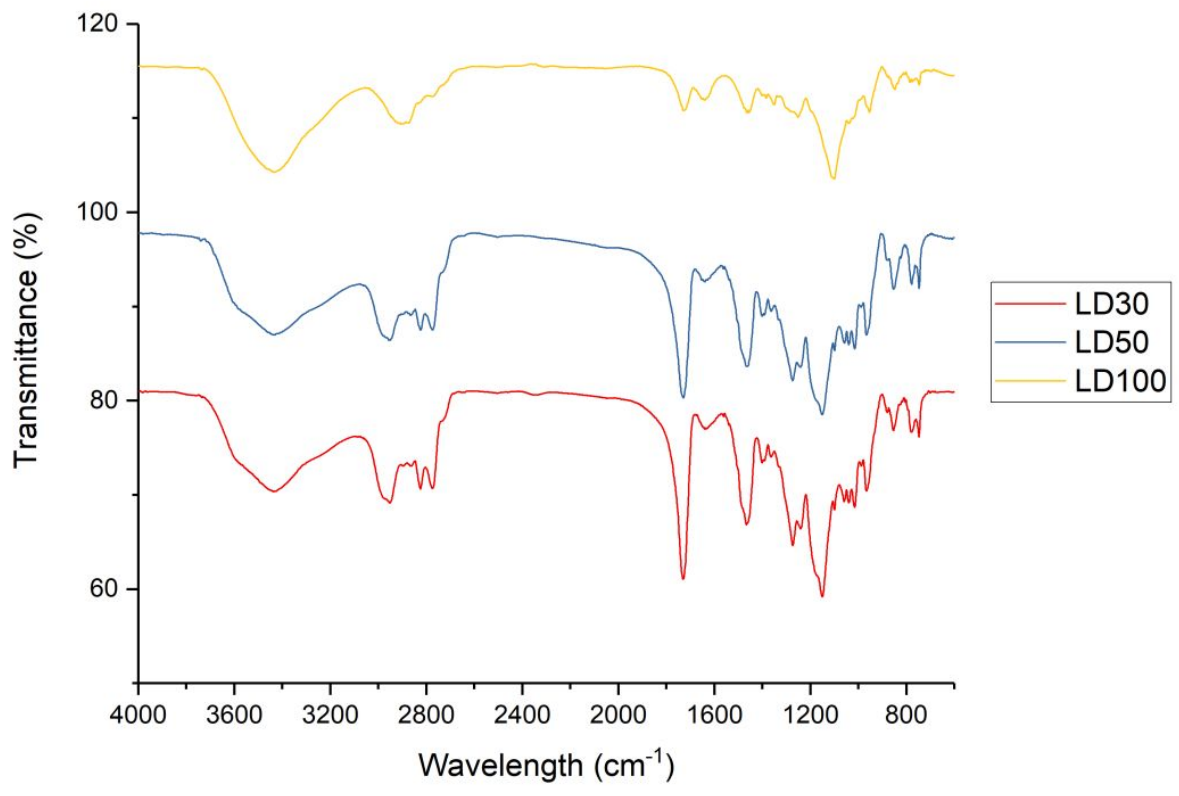

Figure S2: FTIR spectra of lignin-PDMAEMA copolymers which were recorded for 128 scans from 4000 to $600 \mathrm{~cm}^{-1}$ with $1.0 \mathrm{~cm}^{-1}$ resolution 


\section{Cytotoxicity}

Cytotoxicity of the copolymers was evaluated with MTT assay on human dermal fibroblasts. $100 \mu 1$ of DMEM containing a density of 1 X $10^{4}$ cells was added per well of 96-well plate and left to incubate at $37^{\circ} \mathrm{C}$ under $5 \%$ carbon dioxide and $95 \%$ relative humidity atmosphere for 24 h. Subsequently, the culture media were replaced with fresh media containing different copolymers and left to incubate for another $24 \mathrm{~h} .10 \mu \mathrm{lof} 5 \mathrm{mg} / \mathrm{ml}$ sterile-filtered MTT stock solution in phosphate-buffered saline was introduced to each well. The unreacted dye was removed after $4 \mathrm{~h}$ and $100 \mu \mathrm{l}$ of DMSO was added to dissolve the formazan crystals that were formed. Absorbance was measured at $570 \mathrm{~nm}$ using the microplate reader (Infinite M200, Tecan). The cell viability relative to the control containing cells cultured in media without any copolymer was determined by the following equation:

Eq S1: $\quad$ Cell viability $(\%)=\frac{[\text { polymer }]}{[\text { control }]} * 100 \%$

where the tag names represent its corresponding absorbance reading at $570 \mathrm{~nm}$.

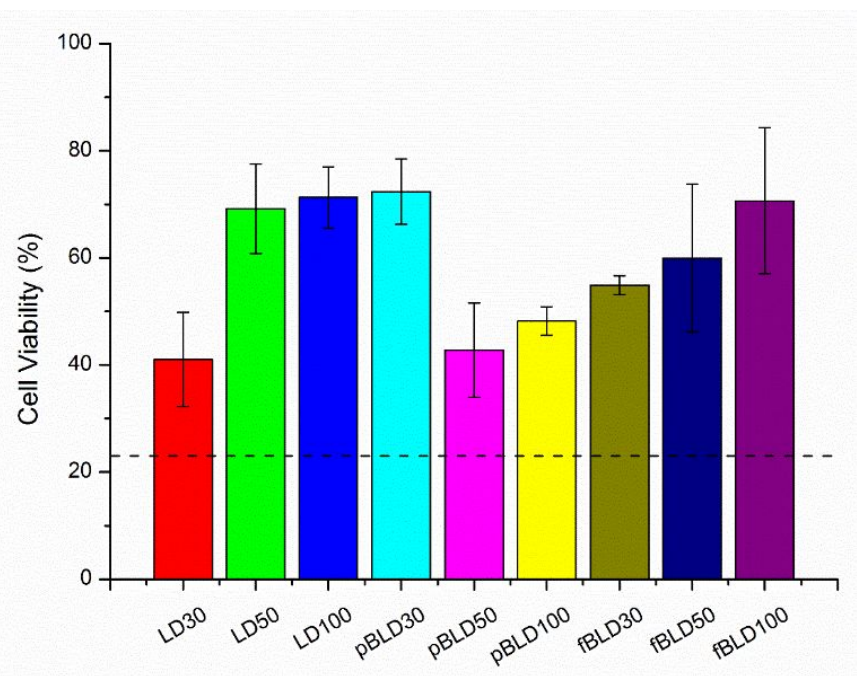

Figure S3: Cell viability of hDFs after $24 \mathrm{~h}$ incubation with lignin-PDMAEMA and quaternized lignin copolymers. The dashed line indicates the cell viability of hDFs after incubated with PDMAEMA homopolymer. 